\title{
环肽中酰胺键 cis-/trans-异构化的相关研究进展
}

\author{
黄 净 $a$ 杨毅华 $*, b$ 冯 娟 $b$ 李军章 $b$ 刘守信*,a \\ ( ${ }^{a}$ 河北科技大学 省部共建国家重点实验室培育基地-河北省药用分子化学重点实验室 河北石家庄 050018) \\ ( ${ }^{b}$ 河北科技大学化学与制药工程学院 河北石家庄 050018)
}

\begin{abstract}
摘要 介绍了环肽化合物中顺式( cis-) 和反式(trans-)结构特点, 分别针对不含 $N$-取代的环肽、含脯氨酸残基的环肽以及 含 $N$-甲基化残基的天然环肽, 讨论了 cis-或 trans-结构在上述环肽及结构类似物中的研究进展, 以及这种 cis-或 trans结构对环肽生物活性的潜在影响. 脯氨酸由于其刚性吡咯环结构而显著影响着环肽分子构象, $N$-甲基化残基不仅增大 了空间位阻，而且能够诱导增大酰胺键 $c i s$-结构比例.
\end{abstract}

关键词 cis-/trans-结构; cis-Itrans-异构体; 环肽; 生物活性

\section{Research Progress on cis-/trans-Isomerization of Cyclic Peptide}

\author{
Huang, Jing ${ }^{a} \quad$ Yang, Yihua $^{*, b} \quad$ Feng, Juan $^{b} \quad$ Li, Junzhang $^{b} \quad$ Liu, Shouxin ${ }^{*, a}$ \\ $\left({ }^{a}\right.$ State Key Laboratory Breeding Base, Hebei Key Laboratory of Molecular Chemistry for Drug, \\ Hebei University of Science \& Technology, Shijiazhuang, Hebei 050018) \\ ( ${ }^{b}$ College of Chemical and Pharmaceutical Engineering, Hebei University of \\ Science \& Technology, Shijiazhuang, Hebei 050018)
}

\begin{abstract}
This paper focuses on cis-/trans-conformational interchanges of amide bonds in cyclic peptides that contain $\mathrm{N}$-unsubstituted amino acids, $\mathrm{N}$-methylated amino acids, and prolines. Conformational preferences of such cyclic peptides and their analogs are discussed. Proline has strong influences on the conformation due to the five-membered cyclic structure. $\mathrm{N}$-Methylation not only increased the steric hindrance, but also led to increased population of cis-conformation of the amide bond.
\end{abstract}

Keywords cis-/trans-structure; cis-/trans-isomerization; cyclic peptide; bioactivity

天然环肽是一类拥有不寻常结构特点和各种生理 活性的化合物 ${ }^{[1]}$. 这类环肽化合物已经超出了有机小分 子的概念，但是它们自身所拥有的立体结构与空间限制 为发现潜在的候选药物提供了独特的选择 ${ }^{[2]}$. 与链状肽 类化合物相比, 环肽化合物通过将链状肽形成环而减少 了无作用的构象. 但环肽分子的构象仍然受制于诸如环 肽分子骨架、分子内氢键、微量金属离子和不同的溶媒 介质等因素的影响, 从而在与蛋白酶作用的过程中, 酶 对环肽的选择性地识别和亲合力 ${ }^{[3]}$ 远远高于链肽, 这些 效应既提高了环肽类化合物治疗疾病的潜力, 同时也因 为提高了环肽化合物代谢的稳定性, 增强了细胞渗透性 和药物分子的口服利用度. 从环肽化合物生物作用的角 度分析, 链肽成环之后的构象, 特别是环肽分子中肽基
酰胺键的 cis-或 trans-结构, 在诱导或控制环肽分子构 象中被视为一个不可忽视的因素, 在生化过程中起到了 关键作用, 尤其是当环肽结构中含有脯氨酸 ${ }^{[4]}$ 片段时, 会引起多肽和蛋白结构的变化, 从而导致多肽生理活性 或蛋白功能的变化, 由此形成的结构或构象特点, 为某 些功能肽和蛋白作用的专一性创造了条件 ${ }^{[5]}$.

\section{1 肽键的 cis-/trans-异构化概述}

对于含有酰胺键结构的化合物, 由于 $\mathrm{N}$ 原子孤对电 子与羰基发生 $\mathrm{p}-\pi$ 共轭作用, 使得酰胺结构中的 $\mathrm{C}-\mathrm{N}$ 单键具有一定程度的双键性质, 其键长 $0.138 \mathrm{~nm}$ 介于 $\mathrm{C}-\mathrm{N}$ 单键 $0.147 \mathrm{~nm}$ 和 $\mathrm{C}=\mathrm{N}$ 双键 $0.128 \mathrm{~nm}$ 之间 ${ }^{[6]}$, 由 此限制了 $\mathrm{C}-\mathrm{N}$ 单键自由旋转, 因此在酰胺、取代氨基

\footnotetext{
* Corresponding authors. E-mail: yihuayang@hebust.edu.cn; chlsx@hebust.edu.cn

Received November 25, 2019; revised January 15, 2020; published online March 11, 2020.

Project supported by the National Basic Research Program of China (Nos. 2011CB512007, 2012CB723501), and the National Natural Science Foundation of China (Nos. 30472074, 30873139).

“973” 计划前期研究专项课题(Nos. 2011CB512007, 2012CB723501)和国家自然科学基金(Nos. 30472074, 30873139)资助项目.
} 
甲酸酯和嫝类等化合物中可能存在顺式 $(c i s-) /$ 反式 (trans-) 异构化现象(图 1). 但是由于这两种异构化之间 的能垒差异不是很大, 通常约为 $10.5 \mathrm{~kJ} / \mathrm{mol}$, 所以这种 cis-/trans-异构化往往不易观察到, 而当酰胺的 $\mathrm{N}$ 原子 上存在空间扭转张力或更大空间位阻时, 这种 cis-/trans-异构化就能十分清楚地显现出来, 如 $N$-甲基 乙酰胺结构中的 cis-/trans-异构化就存在 $83.7 \mathrm{~kJ} / \mathrm{mol}$ 的 旋转能垒差, 二者的 ${ }^{1} \mathrm{H}$ NMR 存在明显差异 ${ }^{[7]}$.

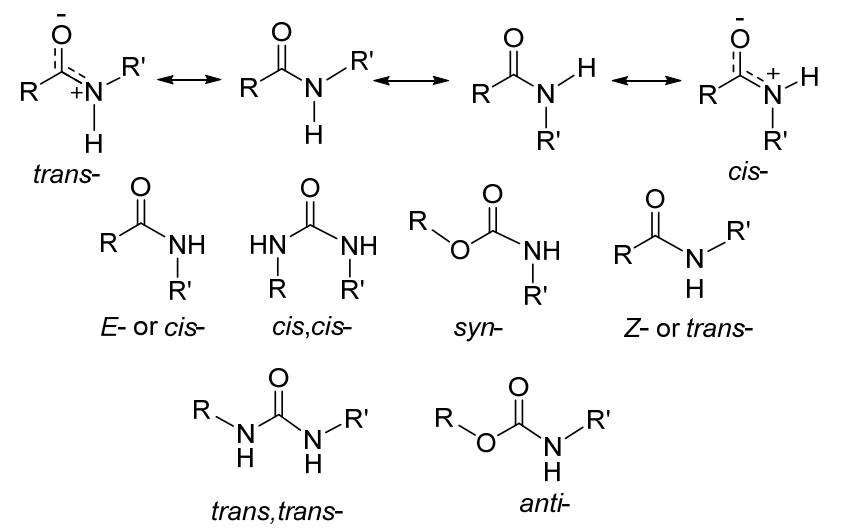

图 1 酰胺键的 cis-/trans-异构化

Figure 1 cis-/trans-Isomerization of amide bond

从生物化学角度来讲, 只有肽类和蛋白质类物质中 的酰胺键才称为肽键, 这种 cis-/trans-异构化的肽基酰 胺键在含有脯氨酸(或吡咯环)残基的各种肽类物质中尤 为常见 ${ }^{[8]}$. 肽链中, 肽基的 cis-/trans-异构化是以肽基酰 胺键为核心的二面角 $\omega\left(\mathrm{C}^{\mathrm{a}}-\mathrm{C}-\mathrm{N}^{+}-\mathrm{C}^{\mathrm{a}}\right)=0^{\circ}$ 时, 定义为 $c i s-$ 结构, 而二面角 $\omega=180^{\circ}$ 时, 定义为 trans-结构. 这个数 值可以有 $\pm 20^{\circ}$ 的波动范围 ${ }^{[9]}$. 在 $2 \mathrm{D}-\mathrm{NMR}$ 核磁共振谱 上, 以肽基脯氨酰胺键为例, 当脯氨酸 $\alpha^{\prime}$-碳原子上 $\alpha^{\prime}-\mathrm{H}$ 与脯氨酸前氨基酸残基 $\alpha$-碳原子上的 $\alpha-\mathrm{H}$ 原子有 $\mathrm{NOE}$ (Nuclear Overhauser Effect)效应时, 这种结构就称为 cis结构; 而当脯氨酸 $\delta^{\prime}$-碳原子上的 $\delta^{\prime}-\mathrm{H}$ 原子与脯氨酸前 残基 $\alpha$-碳原子上的 $\alpha-\mathrm{H}$ 原子有 $\mathrm{NOE}$ 效应时，则称为 trans-结构 ${ }^{[10]}$. 如图 2 所示. 在含有脯氨酸残基的 $c i s$-或 trans-结构中, 由于 trans-异构体的能量仅比 cis-异构体 高出 $2.1 \mathrm{~kJ} / \mathrm{mol}$, 因此 $c i s$-异构体的含量显著增高了 $10 \% \sim 30 \%$, 而且 cis-/trans-异构体的活化能垒差也只有 $54.4 \mathrm{~kJ} / \mathrm{mol}^{[11]}$.

Pook 等 ${ }^{[12]}$ 指出, 处于 $c i s$-结构的脯氨酸残基的 $\beta-\mathrm{CH}_{2}$ 原子受羰基 $\mathrm{C}=\mathrm{O}$ 的屏蔽作用, 相对于 $\gamma-\mathrm{CH}_{2}$ 要 强, 因此, 通过比较脯氨酸残基上 $\mathrm{C}_{\beta}$ 和 $\mathrm{C}_{\gamma}$ 碳谱的化学 位移差值 $\left(\Delta \delta_{\beta \gamma}=\delta_{\beta}-\delta_{\gamma}\right)$, 即可用来判定该肽基脯氨酰胺 键(AA-Pro 或 Pro-AA) 的 cis-或 trans-结构情况, 而且 $c i s-$ 结构的化学位移差值 $\Delta \delta_{\beta \gamma}$ 要比 trans-结构的化学位移差 值大.

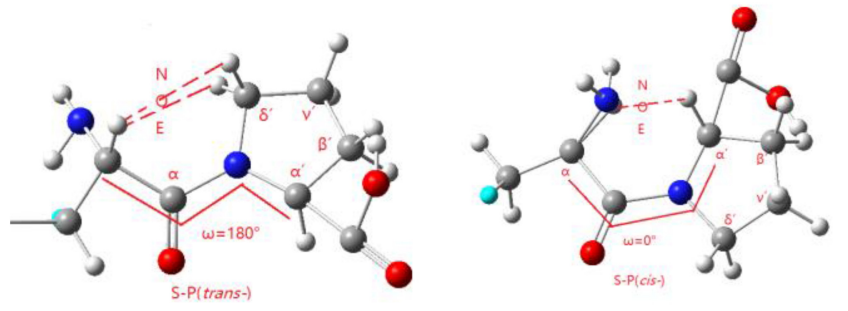

图 2 肽基脯氨酰胺键的 cis-/trans-异构化

Figure 2 cis-/trans-Isomerization of proline-based amide bond

在通常的二肽中, 肽键的 cis-/trans-结构相互转换 势垒不超过 $75 \mathrm{~kJ} / \mathrm{mol}$, 而且室温下转换的速率常数 $<$ $1.0 \mathrm{~s}^{-1}$. 在如此低的旋转势垒下，二肽的肽键通常不会 参与蛋白质的折叠 ${ }^{[13]}$. 然而, 这种 cis-/trans-异构化和 旋转势垒对肽基脯氨酸的影响尤为显著, 而且这种三级 肽键称为肽基脯氨酰胺键. 从热力学角度分析，肽基脯 氨酰胺键的 trans-结构比 cis-结构更为稳定, 因为在 trans-结构中 $\mathrm{C}-\mathrm{N}$ 键两侧基团的空间位阻被完全释放, 对于达到平衡的二级肽键, $c i s$-结构含量约占 $1 \%$. 而对 于三级肽键, 如肽基脯氨酰胺键, 其 $c i s$-和 trans-结构均 存在相近的空间位阻. 因此在非结构蛋白或肽中, cis-或 trans-结构的占比几乎相当，即 cis-异构体和 trans-异构 体含量各接近 $50 \%$. 此外，肽基脯氨酰胺键具有更高的 旋转势垒, 平均活化焓达 $80 \mathrm{~kJ} / \mathrm{mol}$, 而在一般的二肽中 二级肽键的活化焓值不超过 $70 \mathrm{~kJ} / \mathrm{mol}$, 而且肽基脯氨 酰胺键的 cis-/trans-异构化速率常数在 $0.1 \sim 0.01 \mathrm{~s}^{-1}$ 之 间 ${ }^{[13]}$, cis-/trans-异构体转换就相当缓慢, 从而促进了蛋 白质折叠结构的形成.

对于肽键中的 cis-/trans-异构化现象，如果是不含 脯氨酸残基的肽键，目前学术界普遍认为其属于构象异 构, 原因就在于这种 cis-或 trans-异构体在溶液中始终 保持一定的比例共存, 二者无法单独分离, 而且在一定 的条件下可实现 cis-或 trans-异构体相互转化(如升高温 度或改变溶媒). 然而, 当肽键中含有脯氨酸残基或是 $N$-取代的三级肽键时，结合所处的特定环境能够形成稳 定的 cis-或 trans-异构体，这种结构的异构体不仅可以 分离, 而且其 $c i s$-或 $t r a n s$-构型不因外界条件的变化而 改变, 构成事实上的构型异构体.

研究证实, 这种 cis-/trans-异构化在生命现象中发 挥着极为重要的生理功能, 是生命体不可或缺的调控自 身各种生理过程的重要手段. Ramachandran 和 Mitra ${ }^{[11]}$ 通过构象能量计算发现，对于二肽 Ala-Ala(丙氨酸-丙 氨酸)和 Ala-Pro(丙氨酸-脯氨酸), 二者的 cis-异构体所 占比例分别为 $0.1 \%$ 和 $30 \%$ (其对应的焓差分别为 16.7 和 $2.1 \mathrm{~kJ} / \mathrm{mol}$ ). 由于 $c i s$-和 trans-异构体之间能量的差异, 这两者在室温下的相互转化过程就相当缓慢, 但是已经 
证明其对蛋白质的折叠起到了重要作用. 由于这种 cis-/trans-异构化致使小分子在物理、化学乃至生物学性 质方面发生了显著改变, 使得这些具有 cis-/trans-异构 化结构的环肽物质与细胞增值、调亡等生化过程有了重 要的联系. 如药物分子的立体构型和受体之间的相互作 用; 生化过程的立体选择性与分子的立体构型之间的关 系; 各类天然产物的立体构型与其生理活性之间的关系 [14].

酰胺键的 cis-/trans-结构虽然很经典, 但是在环肽 结构与生物活性之间所处的角色一直没有引起关注. 本 文围绕环肽中酰胺键的 cis-/trans-结构, 并按酰胺 $\mathrm{N}$ 原 子上氢是否被取代而分为两大类进行评述. 一类是没有 任何取代的酰胺键 cis-/trans-异构化, 如环化脑啡肽与 皮啡肽中存在的 cis-/trans-异构; 而对取代酰胺键的 cis-/trans-异构, 分为含脯氨酸和 $N$-甲基化残基的两大 类环肽. 应当特别指出的是, $N$-甲基化环肽的生物活性 和口服利用度亦日益受到药物研究的关注 ${ }^{[15]}$.

\section{2 非 $N$-取代环肽的 cis-/trans-异构}

众所周知, 脑啡肽 (Enkephalin, Tyr-Gly-Gly-PheMet 或 Tyr-Gly-Gly-Phe-Leu, Met=蛋氨酸)和皮啡肽 (Dermorphin, Tyr-D-Arg-Phe-Sar, Sar $=N$-甲基甘氨酸)都 属于内源性的阿片肽 (图 3), 具有与吗啡相似的生理功 能. 截止目前为止, 这二者在镇痛方面的应用研究比较 多, 但是镇痛的原理尚未形成共识. 脑啡肽和皮啡肽这

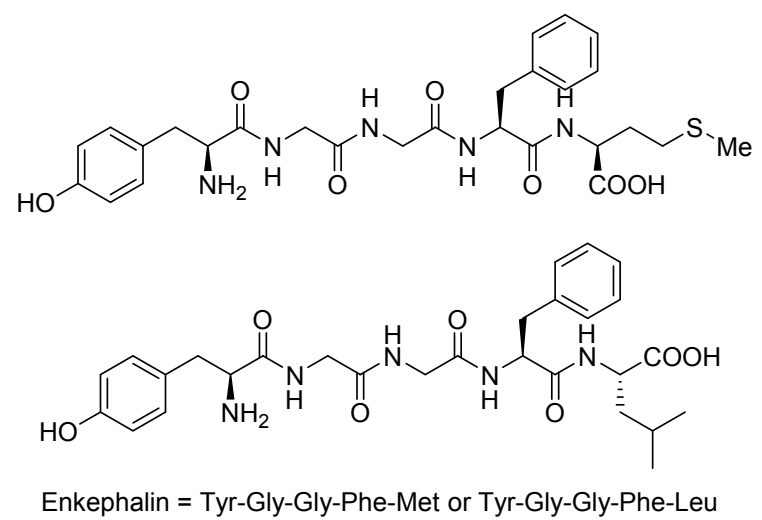<smiles>N=C(N)NCCCC(NC(=O)C(N)Cc1ccc(O)cc1)C(=O)NCC(=O)N(CC(=O)O)c1ccccc1</smiles>

图 3 脑啡肽和皮啡肽的结构

Figure 3 Structures of enkephalin and dermorphin
两种物质由于其链状结构的柔性特点，在遇到不同的受 体位点时可以采取不同的构象形式，从而妨碍了药物化 学家对其关键药理结构特征进行深入研究. 为了限制这 类柔性特点, 一方面可以通过逆向连接和构型翻转来限 制其柔性，另一方面也可以通过分子环化来有效改变柔 性特点. 肽链首尾端的环化不仅降低了线性肽链构象的 灵活性，而且也会产生更为有效的 $\mu$ 或 $\delta$ 受体选择性类 似物。

从化学和生物意义上看, 环肽有别于链肽, 而且具 有更加优良的生物活性. Goodman 等 ${ }^{[16]}$ 在环状脑啡肽类 物质的研究中, 首次合成了系列具有 $c i s$-酰胺键结构的 十四元环肽，而且这些环肽分子中不含有任何脯氨酸或 任何其他 $N$-取代氨基酸残基. 如环肽分子(H-Tyr-c $[D$ Glu-Phe-gPhe- $D$-retroLeu]）（gPhe $=2$-苯基-1,1-二氨基乙 烷, retro=逆向)中的全部酰胺键呈 trans-结构, 而且这 种 trans-异构体的占比达到 $28 \%$; 而含有两个 cis-结构 的异构体占比分别达 $51 \%$ 和 $21 \%$, 即 D-Glu 与 Phe 的酰 胺键呈 cis-结构(占比 51\%), 另一种 Phe 与 $\mathrm{gPhe}$ 的酰胺 键呈 cis-结构(占比 $21 \%$ ). 整个环肽分子在二甲基亚砜 (DMSO) 中同时存在三种 cis-或 trans-异构体, 即 trans-：cis-：cis- $=28 \% ： 51 \% ： 21 \%$ (图 4). 与脑啡肽 (Tyr-Gly-Gly-Phe-Leu) 对比，该环肽化合物在豚鼠回肠 (GPI) 和小鼠输精管(MVD) 的体外活性试验中, 呈现出 较强的 GPI、MVD 效应 ${ }^{[16]}, \mathrm{IC}_{50}$ 值分别达 2.75 和 49.1 $\mathrm{nmol} \cdot \mathrm{L}^{-1}, \mathrm{MVD} / \mathrm{GPI}$ 比值达 17.9 , 即 $\delta / \mu=17.9$, 表明环 化之后具有较高的 $\delta$ 受体选择性.

Schiller 等 ${ }^{[17]}$ 在环状脑啡肽或皮啡肽类似物的研究 中, 通过引入碳碳双键合成了一系列具有 $\mathrm{CH}=\mathrm{CH}$ 双键 结构的环十一、十四肽(图 5).

在合成的环状皮啡肽类似物 I 中，当 4 位为 $L$-构型 的氨基酸残基时，通过烯烃复分解反应( $\mathrm{RCM})$ 反应生成 的双键是 cis-与 trans-结构的混合物; 二者比例为: 化合 物 $1($ cis- $)$ ：化合物 2 (trans- $)=3: 1$; 与此相反, 当 4 位 为 $D$-构型的氨基酸残基时，则仅仅只能得到 $c i s$-结构的 环肽. 与含胱氨酸的母肽 Tyr- $c[D$-Cys-Phe- $(L$ or $D)-\mathrm{Cys}] \mathrm{NH}_{2}$ 相比, 含烯键的环肽在豚鼠回肠(GPI)和小 鼠输精管(MVD)的实验中均显著降低了 $\mu$ 和 $\delta$ 受体激动 效应 ${ }^{[17]}, \mathrm{IC}_{50}$ 值分别只有 436 和 $460 \mathrm{nmol} \cdot \mathrm{L}^{-1}$ (化合物 1 )、 162 和 $444 \mathrm{nmol} \cdot \mathrm{L}^{-1}$ (化合物 2 ), MVD/GPI 的比值表明缺 乏 $\mu$ 、 $\delta$ 受体选择性.

在合成的环状脑啡肽类似物 II 中 ${ }^{[18]}$, GPI 和 MVD 实验表明, 双键 $\mathrm{CH}=\mathrm{CH}$ 处于 trans-结构的环肽在 $\mu 、 \delta$ 受体激动剂和 $\mathrm{k}$ 受体结合力之间存在着平衡作用, $\mathrm{IC}_{50}$ 值分别达 5.63 和 $4.38 \mathrm{nmol} \cdot \mathrm{L}^{-1}$ (化合物 $\mathbf{4}^{\prime}$ ), 其 MVD/GPI 比值约为 0.78 . 


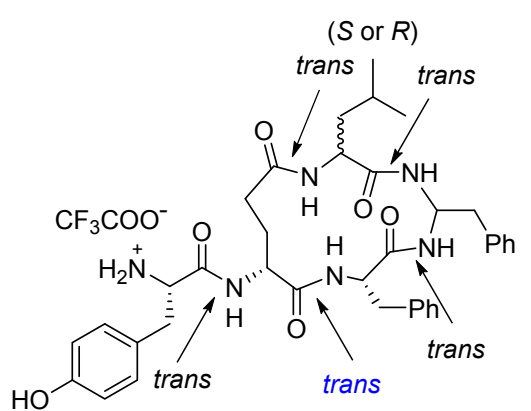

$28 \%$

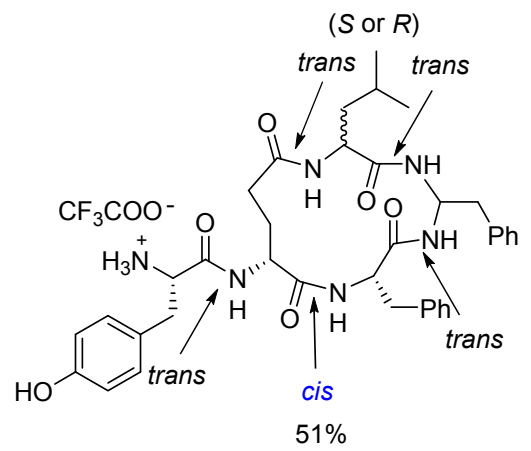

H-Tyr-c[D-Glu-Phe-gPhe-Retro( $S$ or $R)$-Leu]

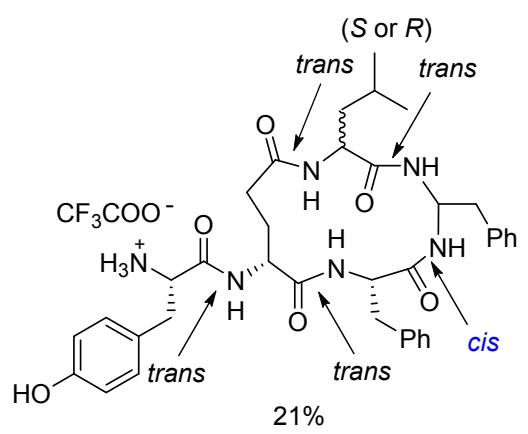

图 4 环状脑啡肽的结构

Figure 4 Structures of cyclic enkephalin

\section{3 含脯氨酸的环肽存在的 cis-/trans-异构}

由于脯氨酸中的氢化吡咯环对环肽分子的构象影 响非常大, 也正是缘于此含有脯氨酸的环肽的生物效应 更加复杂, 目前尚无特定的构效规律可循. 文中所讨论 的含脯氨酸环肽是依据环肽中脯氨酸单元数量的不同, 选取了最为常见的环七～八肽中具有典型 cis-/trans-结 构的环肽为主题来进行讨论.

研究显示 ${ }^{[11,19]}$, 在含脯氨酸的蛋白质类物质中, 有 近 6\%的肽基脯氨基肽键 X-Pro 为 $c i s$-结构, 如脯氨酸脯氨酸(Pro-Pro)、芳香环-脯氨酸(aromatic-Pro)和脯氨 酸-芳香环(Pro-aromatic)序列结构. 肽基脯氨基肽键不 同于其它肽键, 由于氢化吡咯环的刚性结构, 空间位阻 使 $\mathrm{C}-\mathrm{N}$ 酰胺键的双键特性大大降低, 有利于 $\mathrm{C}-\mathrm{N}$ 单 键的自由旋转, 从而形成一定量的 $c i s$-异构体, 而且在

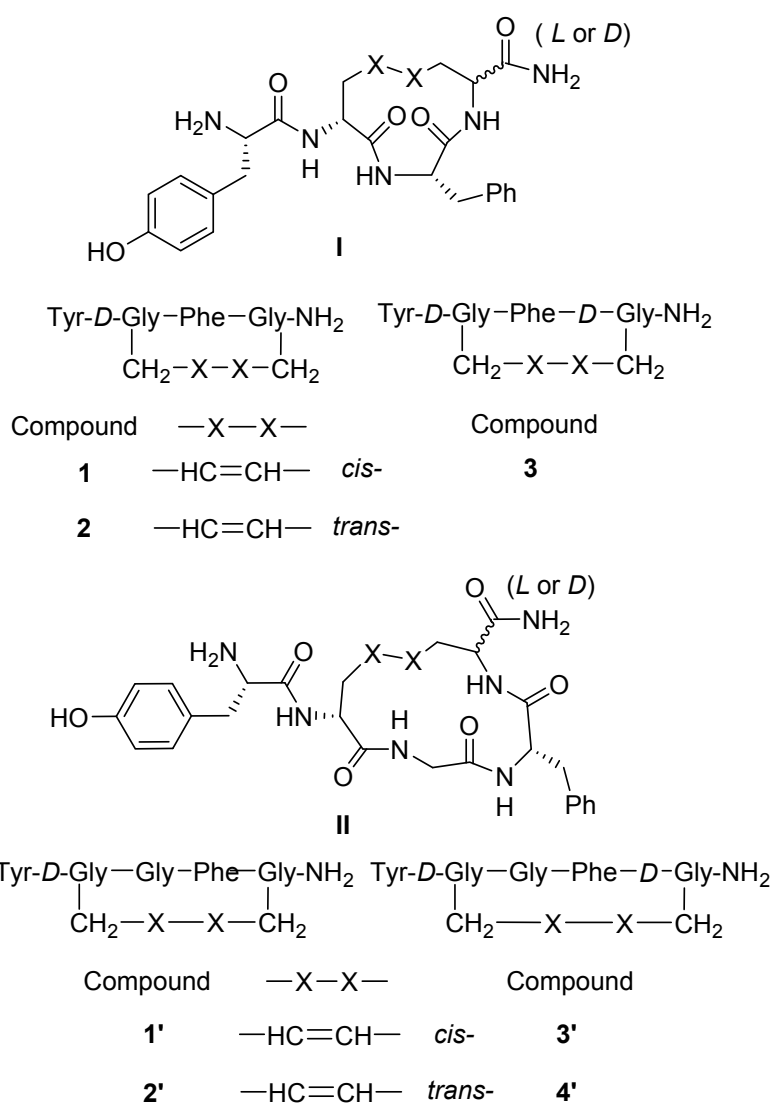

图 5 含 $\mathrm{CH}=\mathrm{CH}$ 双键的环状脑啡肽或皮啡肽类似物 Figure 5 Analogues of cyclic enkephalin or dermorphin containing $\mathrm{C}=\mathrm{C}$ bond

一定的溶剂或 $\mathrm{pH}$ 条件下，能够转变为 $100 \%$ 的 cis-异构 体. 吡咯 $\mathrm{N}$ 原子中 $\mathrm{sp}^{3}$ 杂化轨道上的孤对电子在肽基脯 氨酰胺键的 cis-/trans-异构化中发挥了重要作用，而且 异构体的比例随环肽结构的不同而不同.

\section{1 环七肽 Ceratospongamides 的 cis-/trans-异构}

角状海绵酰胺 Ceratospongamides 是从红藻 Ceratodictyon spongiosum 中分离出来的一种二级代谢产物 ${ }^{[20]}$ (图 6), 其结构中除了一个噻唑、噁唑和两个苯丙氨酸残 基外，还含有两个同属于三级酰胺的脯氨酸残基. 基于 1D-、2D-NMR 核磁共振技术，发现分子中的两个肽基 脯氨酰胺键同时呈 $c i s$-或 trans-结构, 整个分子为具有 cis, cis-或 trans,trans-结构的两种稳定构象的异构体, 即 cis,cis-Ceratospongamide 和 trans,trans-Ceratospongamide 环七肽. 分子模拟研究表明, trans,trans-异构体的整体 分子接近于平面，而 $c i s, c i s$-异构体的分子整体呈更加皱 缩的状态. 生物活性数据显示, trans, trans-异构体能够 显著抑制磷脂酶 $\mathrm{sPLA}_{2}$ 的表达, $\mathrm{ED}_{50}$ 值达 $32 \mathrm{~nm}$, 但是 cis,cis-异构体却没有显现出生物活性.

\section{2 环七肽 Stylostatin 及其类似物中的 cis-/trans-异构}

Stylostatin ${ }^{[21]}$ 是从南太平洋的 Stylotella sp. 海绵中得 

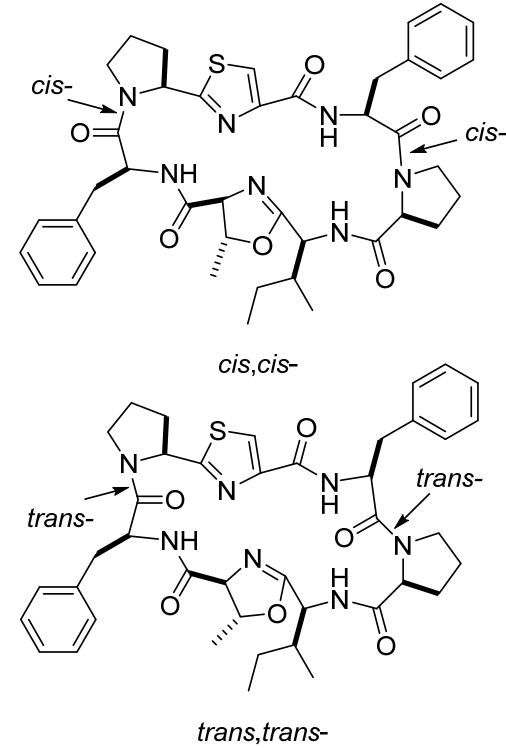

图 6 cis,cis-和 trans,trans-Ceratospongamides

Figure 6 cis,cis- and trans,trans-ceratospongamides

到的一种环七肽, 其结构中的七个氨基酸(脯氨酸、苯丙 氨酸、异亮氨酸、丙氨酸、亮氨酸、丝氨酸和天冬酰胺) 均为 $L$-构型, 而且对小鼠 P388 淋巴细胞性白血病表现 出了良好的抑制效果, $\mathrm{ED}_{50}$ 值达 $0.8 \mu \mathrm{g} / \mathrm{mL}$ (图 7).

通过对五种 Stylostatin 类似物 ${ }^{[22]}$ 的研究显示, 在肽 基脯氨酰胺键 Ile-Pro(异亮氨酸-脯氨酸)的位置容易出 现 cis-或 trans-结构. 核磁共振 NMR 研究显示, 天然环 肽 Stylostatin 中的肽基脯氨酰胺键 $L$-Ile-Pro 呈较为稳定 的 $c i s$-结构. 当用 $D$-别异亮氨酸 ( $D$-allo-Ile) 替换其中的 $L$-异亮氨酸 $(L$-Ile) 时, 则在 $D$-allo-Ile-Pro 的位置形成了 较为稳定的 trans-结构. 而在其余几种结构类似物中, $c i s$-或 trans-结构都呈一定的比例出现, 而且通过改变温 度可以缓慢地实现 cis-或 trans-结构相互转化. 尽管天 然环肽 Stylostatin 与这五种类似物对前列腺肿瘤 (DU-145、LN-caP、SKOV-3)、卵巢肿瘤 (IGROV、 IGROV-ET、SK-BR-3)、黑色素肿瘤(MEL-28)、非小细 胞肺癌(A-549)、肤腺细胞肿瘤(PANC-1、HT-29)、结肠 细胞肿瘤(LOVO、LOVO-DOX)和血癌(K-652)等七种肿 瘤细胞抑制活性不甚突出, 但是由于这种 cis-或 trans结构而导致的大环分子骨架构象上的变化引起了众多 化学工作者的关注.

围绕环肽 Stylostatin 分别引入 $L$-赖氨酸 $(L$-Lys)、 $L$ 精氨酸 $(L-A r g) 、 L$-天冬氨酸 $(L-A s p) 、 L$-天冬酰胺 $(L-A s n)$ 和 $L$-苏氨酸 $(L-T h r)$ 五种天然氨基酸合成了四种结构类 似物(图 7) ${ }^{[23]}$, 通过对肽基脯氨酰胺键 Ile-Pro 的 cis-或 trans-异构化研究显示, 环内氢键和环外氢键都会影响 cis-或 trans-结构的比例及其稳定性，在 DMSO 溶液中, 这四种类似物都存在 cis-或 trans-异构体, 而且 $c i s$-异构 体较 trans-异构体更为稳定.<smiles>[R]C(NC(=O)C(C)NC(=O)[C@H](CC(C)C)NC(=O)[C@H](CO)NC(=O)[C@H](Cc1ccccc1)NC(=O)[C@H](Cc1ccccc1)NC(N)=O)C(=O)N1CCCC1</smiles>

$$
\begin{array}{lc}
\mathrm{R}=L \text {-lle } & \text { cis- or trans- } \\
\mathrm{R}=D \text {-allo-lle } & \text { cis- } \\
\text { trans- }
\end{array}
$$

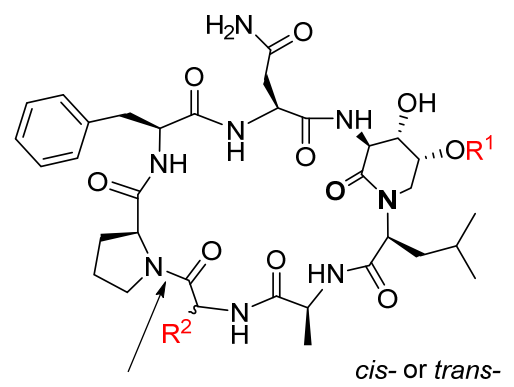

$\mathrm{R}^{1}=\mathrm{Bn}$ or $\mathrm{H} ; \mathrm{R}^{2}=L$-lle cis- or trans$\mathrm{R}^{1}=\mathrm{Bn}$ or $\mathrm{H} ; \mathrm{R}^{2}=D$-allo-lle trans-

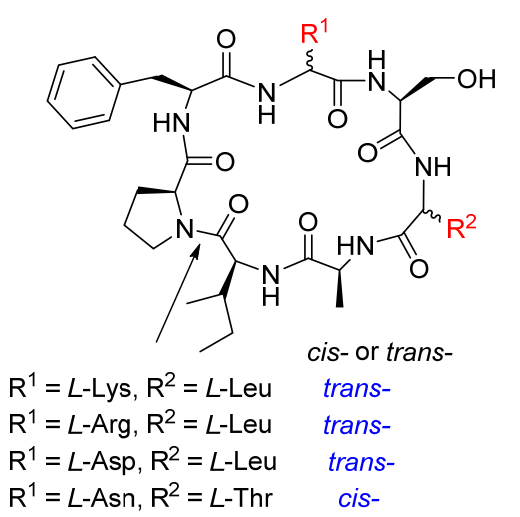

图 7 Stylostatin 及其结构类似物

Figure 7 Stylostatin and its analogues

\section{3 环肽 Sanguinamides 及其类似物中的 cis-/trans-} 异构

Sanguinamides 是从一种 Hexabranchus sanguineus 深红色提取物中分离出来的环肽化合物 ${ }^{[24]}$. 其结构中 除了两个脯氨酸残基和常规的氨基酸残基外，还含有噻 唑或噁唑修饰的氨基酸残基，而且所有氨基酸残基均为 $L$-构型, 并最终确定为环七肽 Sanguinamide A 和环八肽 Sanguinamide B. 最早的研究表明: 在 Sanguinamide A 中两个脯氨酸残基 $\mathrm{Pro}^{1}$ 和 $\mathrm{Pro}^{2}$ 均处于 $c i s$-结构, 而 Sanguinamide B 中则均处于 trans-结构, 即 cis, cis-Sanguinamide $\mathrm{A}$ 和 trans,trans-Sanguinamide $\mathrm{B}$ (简写为 trans, trans-San B). 全合成研究 ${ }^{[25]}$ 显示前者的结构应为 $c i s$, trans-Sanguinamide A (简写为 cis,trans-San A), 如图 8 所 示. 


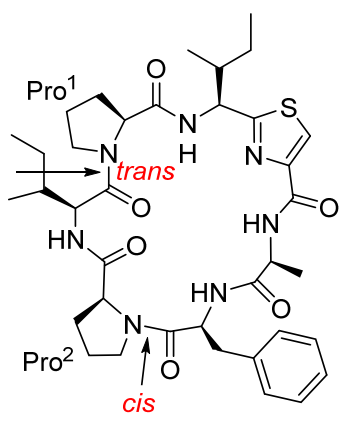

trans, cis-Sanguinamide A

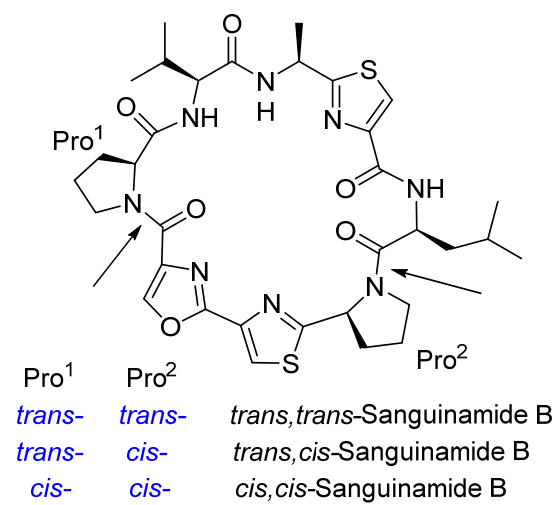

图 8 cis,trans-San A 与 trans,trans-San B 及其异构体

Figure 8 cis,trans-San A, trans,trans-San B and its isomers

通过对 Sanguinamide B 的全合成 ${ }^{[26]}$, 不仅确证了结 构中两个肽基脯氨酰胺键是 trans, trans-结构, 而且还合 成了它的两个异构体, 即 trans, cis-San B 和 cis, cis-San B, 如图 8 所示. 天然环肽 trans,trans-Sanguinamide B 的 trans-结构受热力学控制, 而合成的环肽 cis, cis-San B 的 $c i s$-结构则是受动力学控制. cis, cis-San B 在 DMSO 溶液 中加热至 $170{ }^{\circ} \mathrm{C}$ 回流 $20 \mathrm{~h}$ 可完全转化为 trans,transSanguinamide B 和 trans, cis-San B, 且两者比例为 trans, trans-：trans,cis- $=1 ： 1$. 活性研究显示, 只有 trans, trans-Sanguinamide B 具有破坏铜绿假单胞菌(Pseudomonas aeruginosa) 毒性免疫因子的作用.

McAlpine 通过引入 $N$-甲基化、甘氨酸、 $L$-或 $D$-构 型等结构影响因素, 合成了一系列类似物 ${ }^{[27]}$, 即: trans, $c i s-S a n$ B、cis, cis-San B 和 cis,trans-San B. 结果显示, 只 有引入 $L-/ D$-苯丙氨酸不会导致多种异构体产生, 而引 入 $N$-甲基化氨基酸或甘氨酸等残基的 San B 环肽，都将 生成各种各样的 trans, cis-和 cis, cis-以及 cis,trans-San B 异构体; 即使是在氨基酸序列与手性构型完全相同的情 况下, 也能同时产生两种稳定的 cis,cis-San B 异构体, 如图 9 的环肽 trans,cis-Sanguinamide B 4A 与 trans, cis-Sanguinamide B 4C 两个构型异构体. 所有这些环肽 对革兰氏阳性菌、革兰氏阴性菌、金色葡萄球菌 (Staphylococcus aureus)和大肠杆菌(Escherichia coli) 都
显示出了抑制活性 ${ }^{[27]}$. 构效分析研究发现, 在环肽 $c i s$, $c i s$-San B 的骨架中引入 $L$-或 $D$-苯丙氨酸残基，能够显 著控制大环的构象并赋予大环化合物对细菌运动的最 大抑制作用. 相反, 在该结构模板中引入 $N$-甲基化或甘 氨酸残基, 则无法控制获得构象单一的环肽结构. 所以, 利用活性大环分子的刚性构象特点, 来控制对蛋白质靶 点获得高的亲和力和高的选择性 ${ }^{[28]}$, 可以成为药物设 计和研究的重要手段.

\section{4 环八肽 Phakellistatin 中的 cis-/trans-异构}

Phakellistatins 是从海绵 Phakellia 中提取分离得到 的一类富含脯氨酸残基的环肽类物质，通常由 7 到 10 个氨基酸残基组成，而且至少有一个脯氨酸. 研究结果 表明，其中一些环肽具有癌细胞毒性或抗真菌活性，截 止到目前为止，已经陆续报道了 19 种 Phakellistatin 环 肽 $^{[29]}$.

Phakellistatin 15, Phakellistatin 17 和 Phakellistatin 18 都是从南中国海的海绵 Phakellia fusca 中分离得到三 种环肽 ${ }^{[30]}$ (图 10). 其中, Phakellistatin 15 和 Phakellistatin 17 属于环八肽, 是环肽 Hymenamide $\mathrm{A}$ 的结构类似物 ${ }^{[31]}$, 其结构中含有三个脯氨酸残基，而 Phakellistatin 18 属于 环七肽. 基于 $1 \mathrm{D}$-和 $2 \mathrm{D}-\mathrm{NMR}$ 核磁共振技术, 发现 Phakellistatin 15 和 Phakellistatin 17 两个环肽中的三个脯 氨酸均为 trans-结构, 而 Phakellistatin 18 中的三个脯氨 酸均为 cis-结构. 经体外细胞毒性检测, Phakellistatin 15 具有细胞毒性, 对小鼠白血病细胞 P388 的 $\mathrm{IC}_{50}$ 值达 $8.5 \mu \mathrm{M}$, 而 Phakellistatin 17 和 Phakellistatin 18 对该 P388 细胞和肝癌细胞 BEL-7402 则无细胞毒性.

含有脯氨酸残基的环肽中，酰胺键 cis-/trans-异构 化现象存在的十分普遍，除了上述列举的实例之外，如 环肽 Stylopeptide $1^{[32]} 、$ Euryjanicins ${ }^{[33]} 、$ Carteritins ${ }^{[34]}$ 和 Reniochalistatins ${ }^{[35]}$ 也存在这一现象, 尽管不同环肽中脯 氨酸所扮演的化学异构效应和生物效应不尽相同.

\section{4 含 $N$-甲基氨基酸环肽存在的 cis-/trans-异构}

$N$-甲基化环肽不仅存在于植物和海洋生物体中，也 不断地在发酵液中被分离出来, 所以是发现具有生物活 性环肽的重要途径之一. 笔者课题组 ${ }^{[36]}$ 在围绕环五肽 Sansalvamide A 的研究工作中, 就时常监测到这种 cis-/trans-异构化现象. $N$-甲基化环肽与含有脯氨酸残基 的环肽十分相似, 也存在三级酰胺的 cis-/trans-异构化 现象; 同时，这种 $N$-甲基化环肽的 cis-/trans-结构对环 肽化合物的生理活性影响很大. 如临床上用于抑制器官 移植所引发的排异药物环孢菌素 A (cyclophilin-A)和茜 草科类型环肽 RAs 系列(Rubiaceae-type cyclopeptides, RAs). 


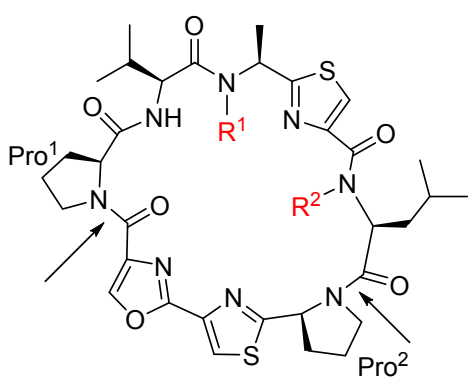

Pro $^{1}$ Pro $^{2}$

$\mathrm{R}^{1}=\mathrm{Me}, \mathrm{R}^{2}=\mathrm{H}, \quad$ trans- $\quad$ cis- $\quad$ trans, cis-San $\mathrm{B} 2 \mathrm{~A}$

$\mathrm{Me} \quad \mathrm{H}$, cis- cis- cis, cis-San $\mathrm{B} 2 \mathrm{~B}$

$\mathrm{H} \quad \mathrm{Me}$, trans- cis- trans, cis-San B 3A

$\mathrm{H} \quad \mathrm{Me}$, cis- cis- cis, cis-San B 3B

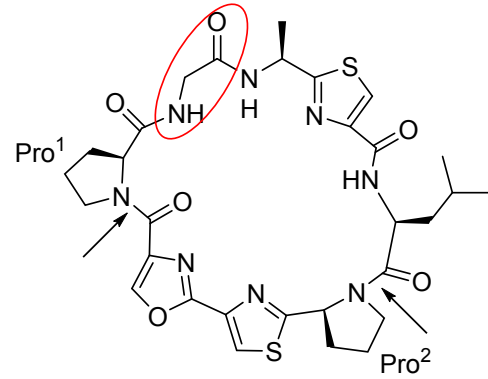

Pro $^{1}$ Pro $^{2}$

trans- cis- trans, cis-San B 4A

cis- cis- cis, cis-San B 4B

trans- cis- trans,cis-San B 4C

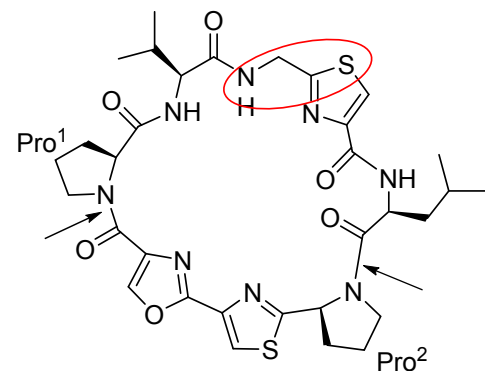

$\mathrm{Pro}^{1} \mathrm{Pro}^{2}$

cis- trans- cis,trans-San B 5A

trans- cis- trans, cis-San B 5B

trans- trans- trans,trans-San B 5C

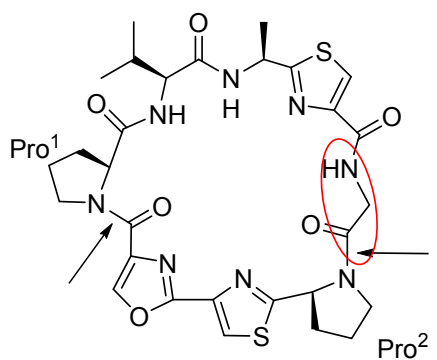

Pro $^{1}$ Pro $^{2}$

trans- cis- cis, trans-San $\mathrm{B} 6 \mathrm{~A}$ trans- trans- trans, cis-San B 6B

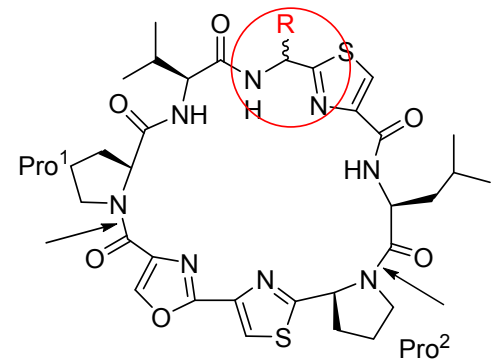

Pro $^{1}$ Pro $^{2}$

$\mathrm{R}=\mathrm{L}-\mathrm{Phe}$ cis- cis- cis,cis-San B 7

D-Phe cis- cis- cis, cis-San B 8

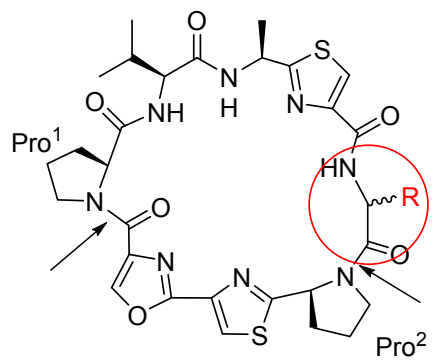

Pro $^{1}$ Pro $^{2}$

$\mathrm{R}=\mathrm{L}-\mathrm{Phe}$ trans- cis- trans, cis-San B 9

D-Phe trans- cis- trans,cis-San B 10

图 9 天然环肽 trans, trans-San B 的 cis-/trans-结构类似物

Figure 9 cis-/trans-Isomeric analogues of trans,trans-San B

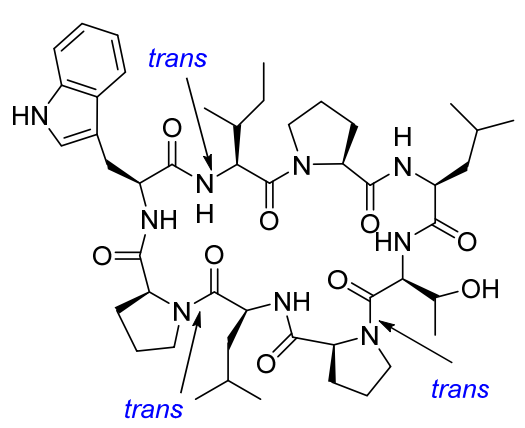

Phakellistatin 15

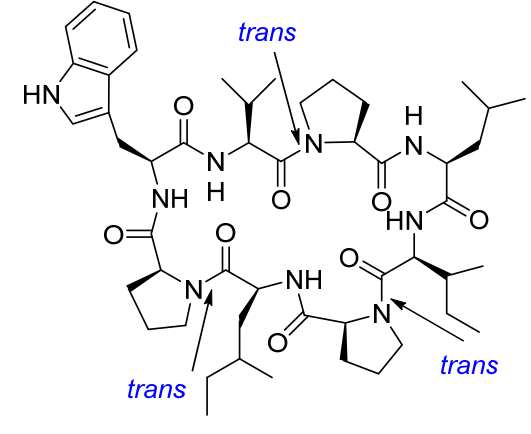

Phakellistatin 17

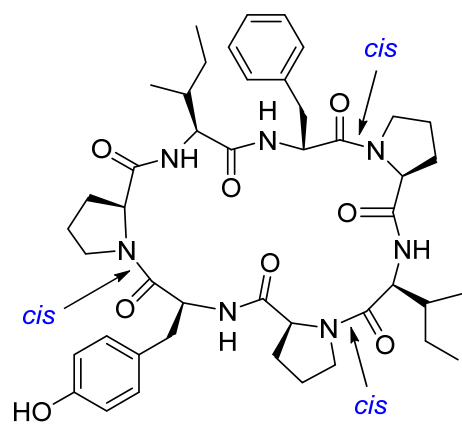

Phakellistatin 18

图 10 Phakellistatins 15、17 和 18 的 cis-/trans-结构

Figure 10 cis-/trans-Isomerization of phakellistatins 15, 17 and 18

\section{1 环孢菌素 A}

环孢菌素 A (Cyclosporine A, CsA ) 亦称 “环孢霉 素” 或 “环孢素”, 是从一种丝状真菌 Tolypocladium inflatum 的培养液中分离出来的环十一肽, 其结构中有 七个 $N$-甲基氨基酸残基，结构如图 11 所示.

最早通过 CsA 单晶研究 ${ }^{[37]}$, 发现整个分子呈扭曲 的 $\beta$-折叠构象, 涉及 $\mathrm{Me}^{-} \mathrm{Val}^{11}, \mathrm{MeBmt}^{1}, \mathrm{Abu}^{2}, \mathrm{Sar}^{3}$, $\mathrm{MeLeu}^{4}, \mathrm{Val}^{5}, \mathrm{MeLeu}^{6}$ 和 $\mathrm{Ala}^{7}$ 八个氨基酸残基, 而且 $\mathrm{MeLeu}^{9}$ 与 $\mathrm{MeLeu}^{10}$ 之间的肽键呈 cis-结构, 整个分子在
氞代丙酮、気代氯仿或気代苯中的分子骨架构象十分相 近，只是在 D-Ala ${ }^{8}$ 区域的溶液或晶体结构稍有不同. 晶 体结构与溶液构象之间的主要差异就在于残基 $\mathrm{MeBmt}^{1}$ 和 $\mathrm{MeLeu}^{10}$ 侧链的取向, 主要是由于 $\mathrm{MeBmt}^{1}$ 侧链羟基 $\mathrm{OH}$ 的分子间氢键在晶体溶解时被破坏所导致; 而且 $\mathrm{MeLeu}^{10}$ 的侧链构象发生改变, 使得 D-Ala ${ }^{8}$ 的分叉氢键 扭曲了这个区域的分子骨架. 直到利用 NMR 技术结合 同位素标记才确定了 CsA 在无水 $\mathrm{THF}$ 溶液中, 残基 $\mathrm{MeLeu}^{9}$ 与 $\mathrm{MeLeu}^{10}$ 之间的肽键呈 $c i s-$ 结构 ${ }^{[38]}$. 

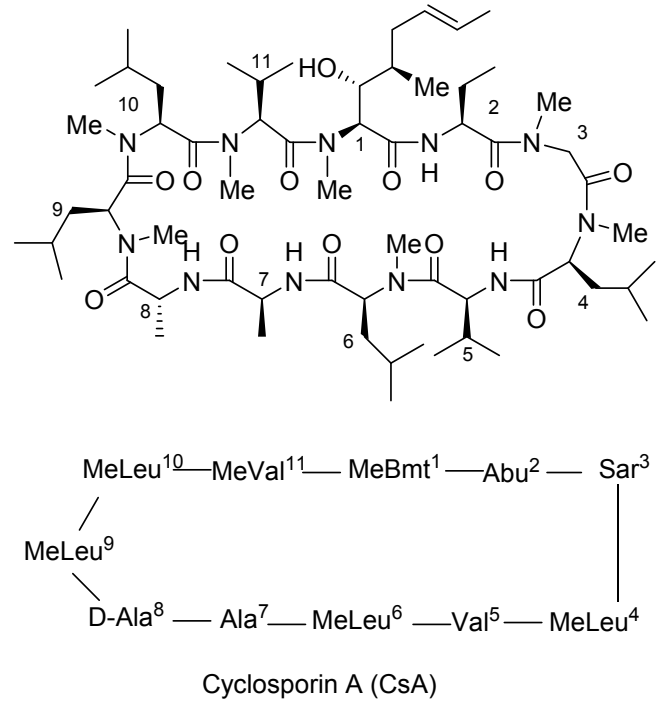

图 11 环孢菌素 A 的结构

Figure 11 Structure of cyclosporine A

CsA 的活性研究表明 ${ }^{[39]}$ :(1) CsA 是亲环蛋白 (cyclophilin)中肽基脯氨基顺-反异构化酶(peptidylprolyl cis-trans isomerase, PPIase)的抑制剂, 其初始抑制活性 和随后的时间依赖性均与溶剂性质有关; (2)在 THF 溶 液中, 残基 $\mathrm{MeLeu}^{9}$ 与 $\mathrm{MeLeu}^{10}$ 之间的 cis-结构对 PPIase 酶没有抑制作用; (3)在 $\mathrm{THF}$ 溶液中添加 $\mathrm{LiCl}$ 或 $\mathrm{CsA}$ 与 亲环蛋白结合后, 残基 $\mathrm{MeLeu}^{9}$ 与 $\mathrm{MeLeu}^{10}$ 之间的 cis结构将转变为对 PPIase 酶有抑制作用的 trans-结构; 4)MeLe ${ }^{9}$-trans-MeLeu ${ }^{10}-\mathrm{MeVal}^{11}$ 呈 trans-结构的残基序 列在亲环蛋白的活性位点上起着高效结合作用, 而相应 的 $c i s-$ 结构则完全不能够被酶识别.

$\mathrm{CsA}$ 与 $\mathrm{LiCl}$ 之间存在络合或不络合两种完全不同 的构象(后者与 $\mathrm{CsA}$ 在氝代氯仿中的构象几乎完全相 同 ${ }^{[40]}$. 尽管与 $\mathrm{LiCl}$ 络合后, 原本残基 $\mathrm{MeLeu}^{9}$ 与 $\mathrm{MeLeu}^{10}$ 之间的 $c i s$-结构转变为 trans-结构, 而且 CsA 中 所有的跨环氢键均被破坏, 使得络合后的 CsA 分子构象 在骨架上存在明显差异, 但仍然同 $\mathrm{CsA}$ 与亲环蛋白受体 结合后的构象非常相似. CsA 在高浓度的 $\mathrm{LiCl}$ 溶液中只 存在一种络合构象.

然而, 在丙酮溶液中的 $\mathrm{CsA}$ 分子构象与络合了 $\mathrm{LiCl}$ 的溶液构象是不同的, 主要差异在于游离的 $\mathrm{CsA}$ 分子中 残基 $\mathrm{MeLeu}{ }^{9}$ 与 $\mathrm{MeLeu}{ }^{10}$ 之间的肽键呈 $c i s$-结构, 而络合 了 $\mathrm{LiCl}$ 后则呈 $\operatorname{trans}$-结构 ${ }^{[41]}$. 当在 $\mathrm{CsA}$ 的 $\mathrm{THF}$ 溶液中 加入 $\mathrm{LiCl}$ 时, $\mathrm{CsA}$ 的分子主骨架肽键全部变为 trans-结 构, 而且这种 trans-结构又是与亲环蛋白结合或是拥有 酶抑制活性的前提条件. 可见, $\mathrm{CsA}$ 分子中 $N$-甲基酰胺 键的结构要受溶剂和是否与 $\mathrm{LiCl}$ 络合的影响. 这一结 果为环孢素药物剂型的确定和制剂方案的制订有重要 的指导意义.

\section{2 茜草科类型环肽 RAs 系列}

茜草科类型环肽 ${ }^{[42]}$ (Rubiaceae-type cyclopeptides, $\mathrm{RAs}$ ) 是具有强抗肿瘤活性的双环六肽，六个氨基酸均 为 $\alpha$-氨基酸, 而且通常由一个 $\mathrm{D}$-丙氨酸(Ala)、一个 $L$ 丙氨酸, 三个 $L-N$-甲基-酪氨酸 $(N-\mathrm{Me}-\mathrm{Tyr})$ 和一个其他类 型的 $L$-氨基酸经肽键依次首尾相连形成十八元环肽，关 键结构特点是两个邻位相连的 $L$-酪氨酸之间的苯环，经 氧桥键形成一个张力较大的十四元环. 茜草科类型环肽 (RAs)因其新颖的结构和显著的抗肿瘤活性而备受关注.

这一类型环肽最早是 1977 年从同种属灌木 Bouvardia ternifolia 中分离得到 Bouvardin 和 Deoxybouvardin (RA-V) ${ }^{[43]}$ 开始，然后从同种属植物 Rubia cordifolia L. 与 Rubia akane 的根或根茎中分离得 到类似的 RA-VII，RA-V，RA-IV 和 RA-III ${ }^{[44]}$ ，以及 RA-I 和 RA-II ${ }^{[45]}$ ，于是将这一系列环肽命名为 RAs 系列. 截止到目前为止, 这一系列植物环肽的数量已 接近三十种 ${ }^{[46]}$, 结构如图 12 所示.

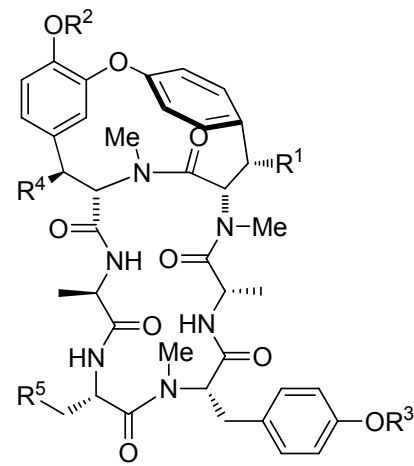

$\begin{array}{llllll}\mathrm{R}^{1} & \mathrm{R}^{2} & \mathrm{R}^{3} & \mathrm{R}^{4} & \mathrm{R}^{5} & \\ \mathrm{OH} & \mathrm{H} & \mathrm{Me} & \mathrm{H} & \mathrm{H} & \text { Bouvardin } \\ \mathrm{H} & \mathrm{H} & \mathrm{Me} & \mathrm{H} & \mathrm{H} & \text { RA-V, Deoxybouvardin } \\ \mathrm{OH} & \mathrm{Me} & \mathrm{Me} & \mathrm{H} & \mathrm{H} & \text { 6-O-Methylbouvardin } \\ \mathrm{H} & \mathrm{H} & \mathrm{Me} & \mathrm{H} & \mathrm{OH} & \text { RA-I } \\ \mathrm{H} & \mathrm{Me} & \mathrm{H} & \mathrm{H} & \mathrm{H} & \text { RA-II } \\ \mathrm{H} & \mathrm{Me} & \mathrm{Me} & \mathrm{H} & \mathrm{OH} & \text { RA-III } \\ \mathrm{H} & \mathrm{Me} & \mathrm{Me} & \mathrm{OH} & \mathrm{H} & \text { RA-IV } \\ \mathrm{H} & \mathrm{Me} & \mathrm{Me} & \mathrm{H} & \mathrm{H} & \text { RA-VII } \\ \mathrm{H} & \mathrm{Me} & \mathrm{Me} & \mathrm{H} & (\mathrm{OH}) \mathrm{Me} & \text { RA-VIII } \\ \mathrm{H} & \mathrm{Me} & \mathrm{Me} & \mathrm{H} & \mathrm{CH}_{2} \mathrm{COOH} & \text { RA-X } \\ \mathrm{H} & \mathrm{H} & \mathrm{Me} & \mathrm{H} & \mathrm{CH}_{2} \mathrm{COOH} & \text { RA-XI } \\ \mathrm{H} & \mathrm{Ac} & \mathrm{Me} & \mathrm{H} & \mathrm{H} & \text { RA-V, O-Acetate }\end{array}$

图 12 茜草科类型双环六肽 RAs 系列

Figure 12 Bicyclic hexapeptide Rubiaceae-type cyclopeptides series

在这一系列中，RA-VII 具有优良的抗肿瘤活性，其 作用机制被认为是通过与真核核糖体的相互作用来抑 制蛋白质的合成 ${ }^{[47]}$. Boger 等 ${ }^{[42]}$ 研究了 RA-VII 的单晶结 构和溶液构象，发现存在三种构象异构体 I、II 和 III，晶 体状态下只存在构象异构体 I, 而且这三种构象异构体 的比例随溶剂性质而改变, 在 THF 溶液中, 三者比例为 $\mathrm{I} ： \mathrm{II}: \mathrm{III}=78: 20: 2$; 在 DMSO 溶液中, 比例为 I： 
II $： I I I=64: 32 ： 4$; 而在氛代氯仿溶液中, 只存在 I 和 II 两种构象异构体, 比例为 $\mathrm{I}: \mathrm{II}=88: 12$. Boger 的研究 表明, RA-VII 分子中三个 $N$-甲基氨基酸残基的 $c i s$-或 trans-结构受溶剂影响变化突出, 只有两个酪氨酸残基 形成的十四元小环上的 $N$-甲基残基始终处于 cis-结构, 而这一 $c i s$-结构又是整个双环六肽分子具有显著生理活 性的关键, 结构如图 13 所示.
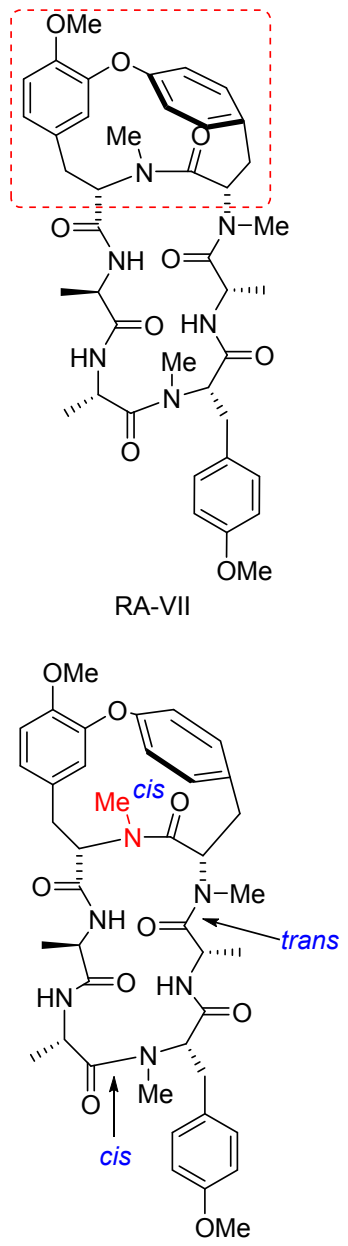

Conformer II
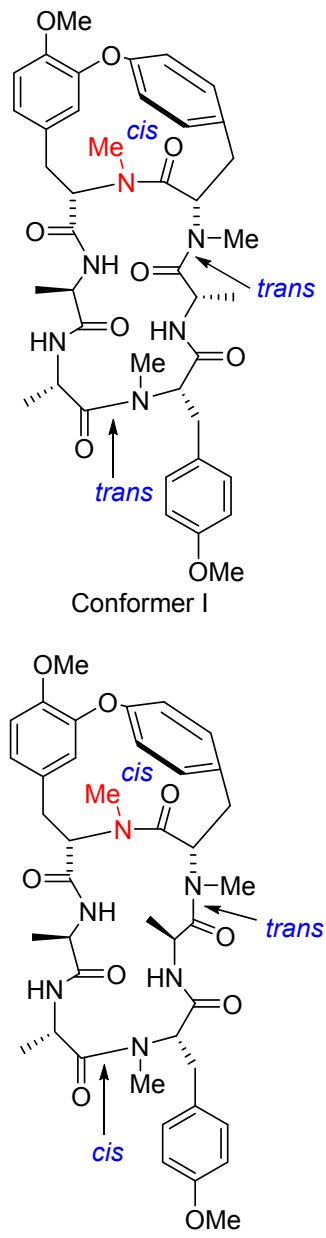

Conformer III
图 13 茜草科类型环肽 RA-VII 及其 cis-/trans-构象异构体

Figure 13 Bicyclic hexapeptide RA-VII and its cis-/trans-isomers

茜草科类型 RAs 系列环肽由于结构新颖和显著的 抗肿瘤活性, 先后有 Inoue, Boger 和祝介平等多个课题 组 ${ }^{[48]}$ 分别围绕二芳基梄构建方法、大环骨架合成、与金 属离子络合效应以及各种构效关系进行了大量研究工 作，而且相关研究还在继续 ${ }^{[49]}$.

近来一种新的环六肽 $O$-seco-RAXXIV ${ }^{[50]}$ 从同种属 植物 Rubia cordifolia L. 中分离得到，毒理实验显示其对 人体早幼粒白血病细胞 $\mathrm{HL}-60$ 有毒理效应, $\mathrm{IC}_{50}=58$ $\mu \mathrm{g} / \mathrm{mL}$ (图 14). 该环肽在氛代甲醇中存在四种 $c i s$-或 trans-异构体, 而且前两种异构体(Conformers I 和 II) 占
比超过 90\%. 即异构体 I 中，残基 $\mathrm{Gln}^{2}$ 与 $\mathrm{Tyr}^{3}$ 之间的 $N$ 甲基化肽键是 $c i s$-结构, 残基 $\mathrm{Ala}^{4}$ 与 $\mathrm{Tyr}^{5}$ 之间的 $N$-甲基 化肽键为 trans-结构, 残基 $\mathrm{Tyr}^{5}$ 与 $\mathrm{Tyr}^{6}$ 之间的 $N$-甲基化 肽键是 cis-结构; 而在异构体 II 中, 则分别依次为 trans-、 trans-和 cis-结构的 $N$-甲基化肽键.
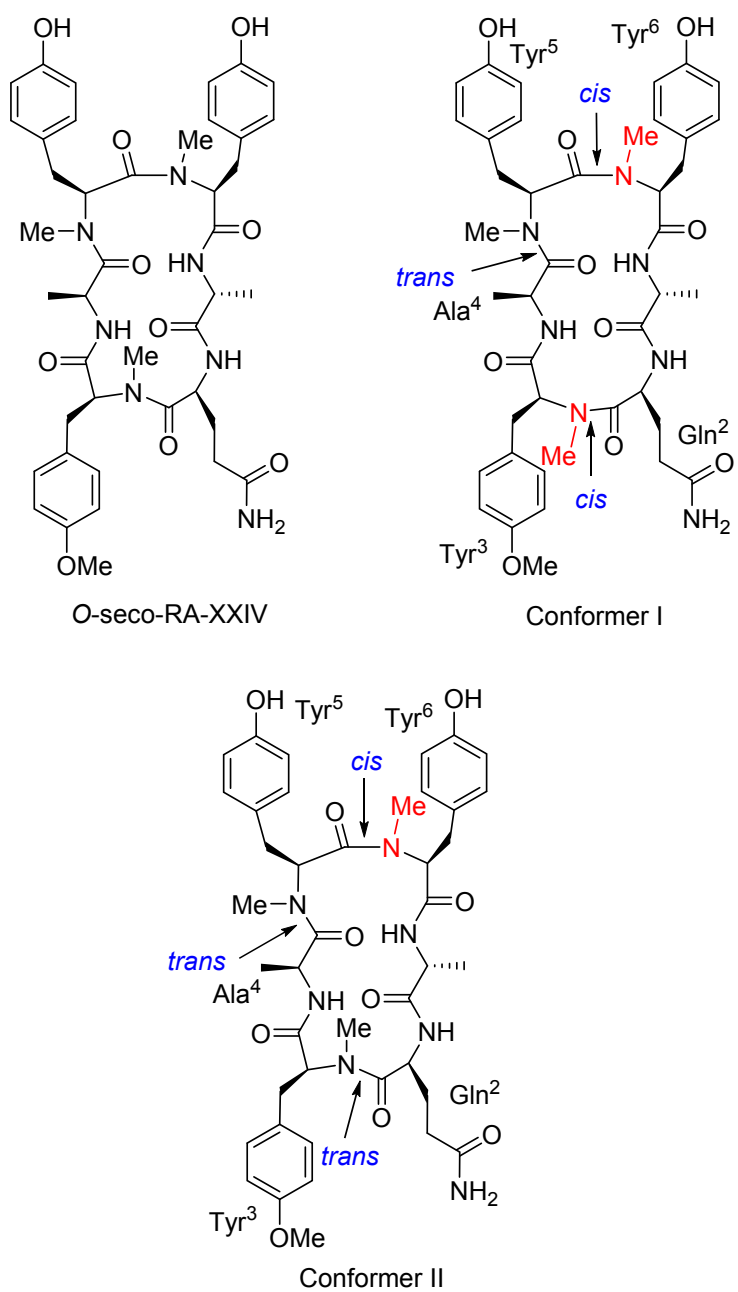

图 14 环六肽 $O$-seco-RA-XXIV 及其 cis-/trans-异构体 Figure 14 Bicyclic hexapeptide $O$-seco-RA-XXIV and its cis-/trans-isomers

谭宁华等 ${ }^{[51]}$ 陆续从茜草科属的各种植物中分离出 来一系列新的环肽化合物, 如 Rubiyunnanins $\mathrm{A} \sim \mathrm{H}$, Rubischumanins $\mathrm{A} \sim \mathrm{C}$, Rubicordins $\mathrm{A} \sim \mathrm{C}$, Rubipodanins $\mathrm{A} \sim \mathrm{B}$, 这些环肽在结构上都与双环六肽 $\mathrm{RAs}$ 相近似, 含有一个或多个 $N$-甲基化氨基酸残基，而且大多显现 出良好的抑制肿瘤细胞生长效应, 特别是环肽 Rubicordins $\mathrm{A} \sim \mathrm{C}$ 和 Rubischumanin $\mathrm{C}$ 中也存在 $N$-甲基 化氨基酸残基的 cis-或 trans-异构体。在这三种 Rubicordins $\mathrm{A} \sim \mathrm{C}$ 环肽中 ${ }^{[52]}$, 每种环肽都存在两个 cis或 trans-异构体(Conformers I 和 II)(图 15). 细胞毒性显 示, Rubicordins A 对胃癌细胞 SGC-7901、肺癌细胞 A-549 和宫颈癌细胞 Hela 都有一定的抑制活性, $\mathrm{IC}_{50}$ 值 
分别为 $2.58 、 20.70$ 和 $3.42 \mu \mathrm{mol} \cdot \mathrm{L}^{-1}(20 \mu \mathrm{g} / \mathrm{mL}$ 浓度 $)$; 同 样地, Rubicordins $\mathrm{B}$ 的 $\mathrm{IC}_{50}$ 值分别为 2.33 、28.74 和 2.57 $\mu \mathrm{mol} \cdot \mathrm{L}^{-1}$; Rubicordins $\mathrm{C}$ 的 $\mathrm{IC}_{50}$ 值分别为 $0.41,1.26$ 和 $0.33 \mu \mathrm{mol} \cdot \mathrm{L}^{-1}$. 可以看出, 环肽 Rubicordins C 对肿瘤细 胞 SGC-7901、A-549 和 Hela 的抑制作用较为突出.
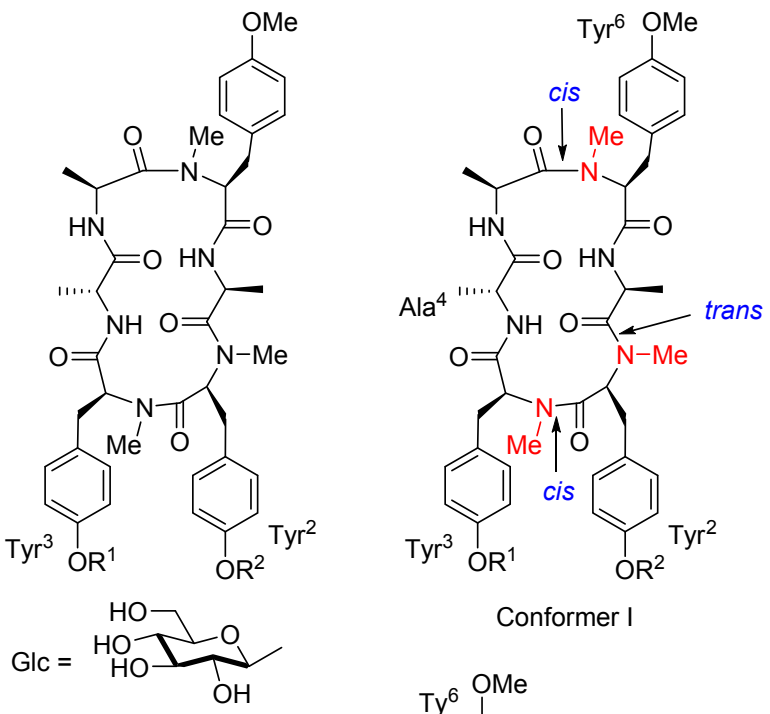

Conformer I

Rubicordins $\mathrm{A} \sim \mathrm{C}$

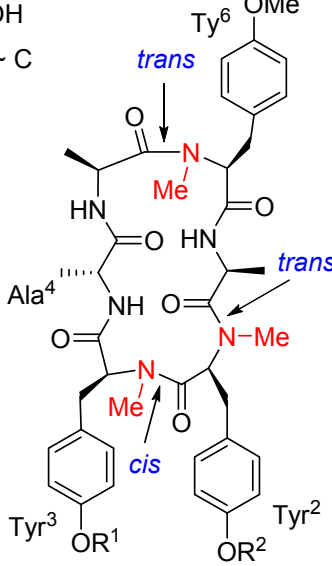

Conformer II

Rubicordin A $\mathrm{R}^{1}=\mathrm{Glc}, \mathrm{R}^{2}=\mathrm{H}, \quad \mathrm{I}: \| \mathrm{I}=64: 36$ Rubicordin $B \quad R^{1}=\mathrm{Glc}, \mathrm{R}^{2}=\mathrm{Me}, \mathrm{I}: \|=58: 42$ Rubicordin $C \mathrm{R}^{1}=\mathrm{Me}, \mathrm{R}^{2}=\mathrm{Me}, \quad \mathrm{I}: \|=52: 48$

图 15 Rubicordins $\mathrm{A} \sim \mathrm{C}$ 及其构象异构体

Figure 15 Rubicordins $\mathrm{A} \sim \mathrm{C}$ and its cis-/trans-isomers

\section{5 结论与展望}

近二十年来, 从海洋珊瑚、藻类、微生物或陆地植 物中发现了许多结构新颖的环肽类化合物, 这些环肽化 合物因具有良好的活性而受到极大关注, 目前已有超过 二十种环肽药物正在进行临床试验 ${ }^{[53]}$. 构效研究显示, 环肽分子中肽基酰胺键的 cis-或 trans-结构通过影响与 DNA 结合的差异而强烈地影响着其生理效应. 尽管从 自然界分离或人工合成的具有明确 $c i s$-或 trans-结构的 环肽种类还很有限，可以预见，在环七～八肽甚至更大
的环肽分子中，无论是含有脯氨酸的酰胺键还是 $N$-甲 基化的酰胺键, cis-/trans-结构限制了分子内跨环氢键作 用而影响整个分子构象，同时 cis-/tran-的比例对溶剂、 温度等条件的依赖性，也会影响着整体分子的构象; 而 对于五肽以下的环肽，因空间限制或很难存在 cis-/tran异构，或因异构化受阻而更易于获得单一异构体，此时 环肽构象控制就更加依赖于氨基酸的手性及其在环肽 序列中的所处位置，故而导致了更为复杂的酰胺键 cis-/trans-结构与生物活性关系. 这些结果对从事相关 研究的化学工作者、药学工作者和生物学工作者有积极 的指导意义和价值.

\section{References}

[1] Jiang, Z.-L.; Hong, J.-L.; Liu, K.-L. Chin. J. Med. Chem. 2004, 14(2), 122 (in Chinese).

(蒋志龙, 郄建坤, 刘克良, 中国药物化学杂志, 2004, 14(2), 122.)

[2] (a) Dougherty, P. G.; Sahni, A.; Pei. D. H. Chem. Rev. 2019, 119 , 10241.

(b) Nielsen, D. S.; Shepherd, N. E.; Xu, W. J.; Lucke, A. J.; Stoermer, M. J.; and Fairlie, D. P. Chem. Rev. 2017, 117, 8094.

[3] (a) Naylor, M. R.; Bockus, A. T.; Blanco, M.; Lokey, R. S. Curr. Opin. Chem. Biol. 2017, 38, 141.

(b) Xin, D. Y.; Jeffries, A.; Burgess, K. ACS Comb. Sci. 2017, 414

[4] Sonti, R.; Gowd, K. H.; Shashanka Rao, K. N.; Ragothama, S.; Rodriguez, A.; Perez, J. J; Balaram, P. Chem. Eur. J. 2013, 19, 15175.

[5] Zondlo, N. J. Acc. Chem. Res. 2013, 46, 1039.

[6] Allen, F. H.; Kennard, O.; Watson, D. G.; Brammer, L.; Orpen, A. G.; Taylor, R. J. Chem. Soc., Perkin Trans. 2 1987, S1.

[7] Smith, M. B.; March, J. Textbook of March's Advanced Organic Chemistry, 5th ed., Trans. by Li, Y.-M., Chemical Industry Publisher, 2009, p. 6 (in Chinese)

(Smith, M. B.; March, J. March，高等有机化学一反应、机理与 结构, 李艳梅译, 第五版, 化学工业出版社, 2009, p. 6.)

[8] Dugave, C.; Demange, L. Chem. Rev. 2003, 103, 2475.

[9] Fischer, G. Angew. Chem., Int. Ed. 1994, 33, 1415.

[10] Galat, A. Curr. Top. Med. Chem. 2003, 3, 1315.

[11] Pal, D.; Chakrabarti, P. J. Mol. Biol. 1999, 294, 271

[12] Siemion, I. Z.; Wieland, T.; Pook, K.-H. Angew. Chem. Int. Ed. 1975, 14, 702 .

[13] Micheau, J. C.; Zhao, J. Z. J. Phys. Org. Chem. 2007, 20, 810

[14] (a) Cordato, D. J.; Mather, L. E.; Herkes, G. K.; Mather, L. E.; Morgan, M. K. J. Clin. Neurosci. 2003, 10(6), 649.

(b) Hu, G. P.; Yuan, J.; Sun, L.; She, Z. G.; Wu, J. H.; Lan, X. J.; Zhu, X.; Lin, Y. C.; Chen, S. P. Mar. Drugs 2011, 9, 514.

(c) Yi, P.; Li, Y.-X.; Zhang, M.-M.; Wang, Y.; Ye, B.-P. Pharm. Biotechnol. 2014, 21(3), 264 (in Chinese).

(伊鹏; 李英新; 张萌萌; 王颖; 叶波平，药物生物技术，2014， 21(3), 264.)

[15] Räder, A. F. B.; Reichart, F.; Weinmüller, M.; Kessler, H. Bioorg. Med. Chem. 2018, 26, 2766.

[16] (a) Mierke, D. F.; Yamazaki, T.; Said-Nejad, O. E.; Felder, E. R.; Goodman, M. J. Am. Chem. Soc. 1989, 111, 6847.

(b) Said-Nejad, O. E.; Felder, E. R.; Mierke, D. F.; Yamazaki, T.; Schiller, P.; Goodman, M. Int. J. Pept. Protein Res. 1992, 39, 145.

[17] Berezowska, I.; Chung, N. N.; Lemieux, C.; Wilkes, B. C.; Schiller, P. W. Acta Biochim. Pol. 2006, 53(1), 73.

[18] Berezowska, I.; Chung, N. N.; Lemieux, C.; Wilkes, B. C.; Schiller P. W. J. Med. Chem. 2007, 50, 1414.

[19] Jabs, A.; Weiss, M. S.; Hilgenfeld, R. J. Mol. Biol. 1999, 286, 291.

[20] Tong, T. L.; Williamson, R. T.; William, H. G. J. Org. Chem. 2000 , 65,419 . 
[21] Pettit, G. R.; Srirangam, J. K.; Herald, D. L.; Erickson, K. L.; Doubek, D. L.; Schmidt, J. M.; Tackett, L. P.; Bakudc, G. J. J. Org. Chem. 1992, 57, 7217.

[22] Forns, P.; Piro, J.; Cuevas, C.; Garcia, M.; Rubiralta, M.; Giralt, E.; Diez, A. J. Med. Chem. 2003, 46, 5825.

[23] Lopez-Martinez, C.; Flores-Morales, P.; Cruz, M.; Gonzalez, T.; Feliz, M.; Diez, A.; Campanera, J. M. Phys. Chem. Chem. Phys. 2016, $18,12755$.

[24] Dalisay, D. S.; Rogers, E. W.; Edison, A. S.; Molinski, T. F. J. Nat. Prod. 2009, 72, 732 .

[25] Nielsen, D. S.; Hoang, H. N.; Lohman, Rink-Jan; Diness, F.; Fairlie, D. P. Org. Lett. 2012, 14, 5720.

[26] Singh, E. K.; Ramsey, D. M.; McAlpine, S. R. Org. Lett. 2012, 14, 1198.

[27] (a) Wahyudi, H.; Tantisantisom, W.; Liu, X.; Ramsey, D. M.; Singh, E. K.; McAlpine, S. R. J. Org. Chem. 2012, 77, 10596.

(b) Wahyudi, H.; Tantisantisom, W.; McAlpine, S. R. Tetrahedron Lett. 2014, 55, 2389.

(c) Pietkiewicz, A. L.; Wahyudi, H.; McConnell, J. R.; McAlpine, S. R. Tetrahedron Lett. 2014, 55, 6979.

[28] Chatterjee, J.; Mierke, D.; Kessler, H. J. Am. Chem. Soc. 2006, 128, 15164.

[29] Marta, P. G.; Alessandra, M.; Judit, T. P.; Fernando, A. J. Med. Chem. 2013, 56, 9780

[30] Zhang, H. J.; Yi, Y. H.; Yang, G. J.; Hu, M. Y.; Cao, G. D.; Yang, F.; Lin, H. W. J. Nat. Prod. 2010, 73, 650 .

[31] Kobayashi, J. I.; Tsuda, M.; Nakamura, T.; Mikami, Y.; Shigemori, H. Tetrahedron 1993, 49, 2391.

[32] Pettit, G. R.; Srirangam, J. K.; Herald, D. L.; Xu, J.; Boyd, M. R.; Cichacz, Z.; Kamano, Y.; Schmidt, J. M.; Erickson, K. L. J. Org. Chem. 1995, 60, 8257.

[33] (a) Vera, B.; Vicente, J.; Rodriguez, A. D. J. Nat. Prod. 2009, 72, 1555 .

(b) Aviles, E.; Rodriguez, A. D. Tetrahedron 2013, 69, 10797.

[34] Afifi, A. H.; El-Desoky, A. H.; Kato, H.; Mangindaan, R. E. P.; Voogd, N. J. de; Ammar, N. M.; Hifnawy, M. S.; Tsukamoto, S. Tetrahedron Lett. 2016, 57, 1285.

[35] Zhan, K. X.; Jiao, W. H.; Yang, F.; Li, J.; Wang, S. P.; Li, Y. S.; Han, B. N.; Lin, H. W. J. Nat. Prod. 2014, 77, 2678.

[36] (a) Gu, W.; Liu, S. X.; Silverman, R. B. Org. Lett. 2002, 4, 4171. (b) Liu, S..; Feng, X.; Y. Geng,; Yao, J.; Li, J. Chin. J. Org. Chem. 2005, 25, 604 (in Chinese).

(c) Liu, S.; Gu, W.; Lo, D.; Ding, X.; Ujiki, M.; Adrian, T. E.; Soff, G. A.; Silverman, R. B. J. Med. Chem., 2005, 48, 3630 .

(d) Liu, S.; Yang, Y.; Zhao, C.; Huang, J.; Han, C.; Han, J. Med. Chem. Commun. 2014, 5, 463.

[37] Loosli, H. R.; Kessler, H.; Oschkinat, H.; Weber, Ha. P.; Petcher, T. J.; Widmer, A. Helv. Chim. Acta 1985, 68, 682.

[38] Fesik, S. W.; Gampe, R. T.; Holzman, T. F.; Egan, D. A.; Edalji, R.; Luly, J. R.; Simmer, R.; Helfrich, R.; Kishore, V.; Rich, D. H. Science 1990, 250, 1406.

[39] Kofron, J. L.; KuzmiE, P.; Kishore, V.; Gemmecker, G.; Fesik, S. W.; Rich, D. H. J. Am. Chem. Soc. 1992, 114, 2670.

[40] Kock, M.; Kessler, H.; Seebach, D.; Thaler, A. J. Am. Chem. Soc. 1992, 114, 2676.

[41] Verheyden, P.; Jaspers, H.; Wolf, E. D.; Binst, G. V. Int. J. Peptide Protein Res. 1994, 44, 364.

[42] Boger, D. L.; Michael, A. P.; Zhou, J. C. J. Am. Chem. Soc. 1995, $117,7357$.
[43] Jolad, S. D.; Hoffmann, J. J.; Torrance, S. J.; Wiedhopf, R. M.; Cole, J. R.; Arora, S. K.; Bates, R. B.; Gargiulo, R. L.; Kriek, G. R. J. Am. Chem. Soc. 1977, 99, 8040.

[44] Itokawa, H.; Takeya, K.; Mihara, K.; Mori, N.; Hamanaka, T.; Sonobe, T.; Iitaka, Y. Chem. Pharm. Bull. 1983, 31, 1424.

[45] Itokawa, H.; Takeya, K.; Mori, N.; Sonobe, T.; Mihashi, S.; Hamanaka, T. Chem. Pharm. Bull. 1986, 34, 3762.

[46] (a) Tan, N. H.; Zhou, J. Chem. Rev. 2006, 106, 840.

(b) Morita, H.; Takeya, K. Heterocycles 2010, 80, 739.

(c) Zhao, S. M.; Kuang, B.; Fan, J. T.; Yan, H.; Xu, W. Y.; Tan, N. H. Chimia 2011, 65, 952.

[47] Lee, E. J.; Hitotsuyanagi, Y.; Nakagawa, Y.; Kato, S.; Fukaya, H.; Takeya, K. Bioorg. Med. Chem. Lett. 2008, 18, 6458.

[48] (a) Inaba, T.; Umezawa, I.; Yuasa, M.; Inoue, T.; Mihashi, S.; Itokawa, H.; Ogura, K. J. Org. Chem. 1987, 52, 2957.

(b) Itokawa, H.; Morita, H.; Takeya, K. Chem. Phar. Bull. 1992, 40, 1050 .

(c) Inoue, T.; Inaba, T.; Umezawa, I.; Yuasa, M.; Itokawa, H.; Oaura, K.; Komatsu, K.; Hara, H.; Hoshino, O. Chem. Pharm. Bull. $\mathbf{1 9 9 5}, 43,1325$.

(d) Boger, D. L.; Yohannes, D. J. Am. Chem. Soc. 1991, 113, 1427.

(e) Boger, D. L.; Yohannes, D.; Zhou, J.; Patane, M. A. J. Am. Chem. Soc. 1993, 115, 3420.

(f) Boger, D. L.; Patane, M. A.; Zhou, J. J. Am. Chem. Soc. 1994, 116,8544 .

(g) Boger, D. L.; Patane, M. A.; Zhou, J. J. Am. Chem. Soc. 1995, $117,7357$.

(h) Boger, D. L.; Zhou, J. C. J. Am. Chem. Soc. 1995, 117, 7364.

(i) Beugelmans, R.; Bigot, A.; Bois-Choussy, M.; Zhu, J. P. J. Org. Chem. 1996, 61, 771.

[49] (a) Hitotsuyanagi, Y.; Odagiri, M.; Kato, S.; Kusano, J.; Hasuda, T.; Fukaya, H.; Takeya, K. Chem.-Eur. J. 2012, 18, 2839.

(b) Lee, J. E.; Hitotsuyanagi, Y.; Takeya, K. Tetrahedron 2008, 64, 4117.

(c) Lee, J. E.; Hitotsuyanagi, Y.; Kim, I. H.; Hasuda, T.; Takeya, K. Bioorg. Med. Chem. Lett. 2008, 18, 808.

(d) Lee, J. E.; Hitotsuyanagi, Y.; Fukaya, H.; Kondo, K.; Takeya, K. Chem. Pharm. Bull. 2008, 56, 730 .

(e) Hitotsuyanagi, Y.; Ishikawa, H.; Hasuda, T.; Takeya, K. Tetrahedron Lett. 2004, 45, 935.

(f) Hitotsuyanagi, Y.; Hasuda, T.; Matsumoto, Y.; Yamaguchi, K.; Itokawa, H.; Takeya, K. Chem. Commun. 2000, 1633.

[50] Hitotsuyanagi, Y.; Kusano, J. I.; Kim, I. H.; Hasuda, T.; Fukaya, H.; Takeya, K. Phytochem. Lett. 2012, 5, 335.

[51] (a) Fan, J. T.; Chen, Y. S.; Xu, W. Y.; Du, L. Ch.; Zeng, G. Z.; Zhang, Y. M.; Su, J.; Li, Y.; Tan, N. H. Tetrahedron Lett. 2010, 51, 6810 .

(b) Fan, J. T.; Su, J.; Peng, Y. M.; Li, Y.; Li, J.; Zhou, Y. B.; Zeng, G. Z.; Yan, H.; Tan, N. H. Bioorg. Med. Chem. 2010, 18, 8226.

(c) Huang, M. B.; Zhao, S. M.; Zeng, G. Z.; Kuang, B.; Chen, X. Q.; Tan, N. H. Tetrahedron 2014, 70, 7627.

(d) Wang, Z.; Zhao, S. M.; Zhao, L. M.; Chen, X. Q.; Zeng, G. Z.; Tan, N. H. PLoS One 2015, 10, e0144950.

(e) Hu, Y. Y.; Feng, L.; Wang, J.; Zhang, X. J.; Wang, Z.; Tan, N. H. Chem. Biodivers. 2019, 16(1), e1899438.

[52] Chen, X. Q.; Zhao, S. M.; Wang, Z; Zeng, G. Z.; Huang, M. B.; Tan, N. H. Tetrahedron 2015, 71, 9673.

[53] Abdalla, M. A.; McGaw, L. J. Molecules 2018, 23, 2080.

(Li, L.; Fan, Y.) 